\title{
Characterization of the shape of gold nanoparticles prepared by thermal annealing
}

\author{
Attila Bonyár, Judit Kámán \\ Department of Electronics Technology \\ Budapest University of Technology and Economics \\ Budapest, Hungary \\ bonyar@ett.bme.hu
}

\author{
István Csarnovics, László Balázs \\ Department of Experimental Physics \\ University of Debrecen \\ Debrecen, Hungary
}

\begin{abstract}
Gold nanoparticles - which are intended to be used as transducers in a localized surface plasmon resonance (LSPR) sensor - were prepared by thermally annealing various layers of gold thin films deposited on glass substrate. The size and distribution of nanoparticles were investigated by atomic force microscopy (AFM). The changes in the nanoparticle shape in function of the deposition and annealing parameters are characterized. A novel parameter called localization factor was used to investigate the shape of the resulting particles. A common problem concerning the AFM imaging of nanoparticles, namely the tip convolution effect was studied, and possibilities to use the localization factor parameter to optimize surface reconstruction algorithms via tip deconvolution is demonstrated.
\end{abstract}

Keywords - nanometrology, gold nanoislands, localization factor, imaging artifacts, $A F M$

\section{INTRODUCTION}

Gold nanoparticles are widely used as signal amplification elements in various electrochemical and optical sensor applications [1]. This includes for example the conjugation of $\mathrm{Au}$ NPs with biomolecules as biological tags in biosensoric application, but Au NP modified sensor transducer surfaces (e.g. thin films) are also quite frequent. The nanoparticles on the transducer surface can in one hand increase the effective electrode area in electrochemical applications, while in optical sensors the so called localized surface plasmon resonance (LSPR) phenomenon - the collective oscillations of delocalized electrons in response to an external electric field - is utilized to amplify the electric near field and thus the sensor sensitivity.

Au NPs can be synthesized in several ways [2]. Perhaps one of the simplest methods is the thermal annealing of predeposited gold thin films on glass or silicon surfaces. In this method the parameters of the annealing process (time, temperature) and the pre-deposited thin film thickness influence and define the resulting size and distribution of the Au NPs on the surface [3]. While the size and distribution of the resulting particles can be easily measured with AFM, the shape of the prepared nanoparticles are harder to characterize. For this reason a novel parameter called localization factor is to be used.

The localization factor parameter was introduced for the characterization of AFM images by Bonyár in 2012 [4]. This parameter can be obtained by calculating the so called generalized localization (which is the structural entropy and spatial filling factor function pair) of an AFM image, and it is meant to characterize the typical shape of the surface structures. Since its introduction the possible application of the localization factor was demonstrated for the characterization of gold thin film microstructures [4]; for the shape change of gold grains during thermal annealing [5]; and also for the detection of tin oxidation and oxide grain formation [6]. Now we intend to demonstrate the possible use of this parameter for the characterization of the shape of gold nanoparticles prepared by thermal annealing.

A common problem during AFM imaging originates from the very principles of using a scanning probe to measure the topography of surfaces, namely from the geometrical convolution of the tip and the surface structures. As illustrated in Fig. 1, the difference between the obtained structures imaged with a sharp tip and a blunt tip (caused by either damage, wear or contamination) can be significant, especially in the case of sharp surface features. This problem is common with nanoparticles since they have both small size and high contact angle at their sides. The amount of structure dilation experienced while measuring small nanoparticles is strongly depending on the quality of the tip. There are built-in algorithms in most AFM image post-processing software to handle this issue. It is possible to estimate the geometry of the used tip based on the image, and it is also possible to try to reconstruct the image, by using deconvolution with the assessed tip geometry. The two problems with such algorithms are that 1) it is not possible to perfectly reconstruct an image due to the loss of information at the convoluted areas; and 2) they are strongly depending on the parameters used for tip assessment. In this work we aim to demonstrate, that the localization factor parameter can be used to optimize the surface reconstruction process.

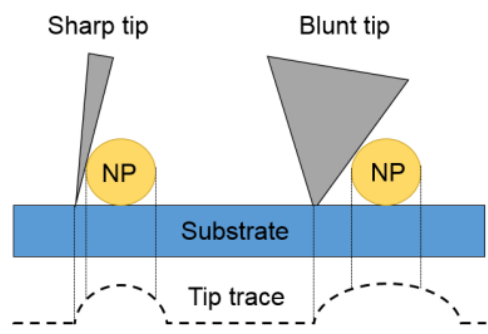

Fig. 1. Illustration of the geometrical convolution and the widening of features with sharp and blunt AFM tips. 


\section{THEORY}

In this section we discuss the necessary mathematical background to understand the localization factor parameter. For the detailed description of this parameter see our original articles regarding its introduction for the characterization of thin film surfaces $[4,6]$.

To calculate the localization factor one must calculate the generalized localization of an AFM image. The generalized localization can be derived from the structural entropy $\left(S_{s t r}\right)$ and the spatial filling factor $(q)$ functions of the image. Since the intensity distribution $\left(I_{i}\right)$ of a contact-AFM image can be normalized in a way to fulfill equations (1) and (2), the image can be well characterized with the structural entropy and the spatial filling factor.

$$
\begin{gathered}
Q_{i} \geq 0 \text { for } i=1, \ldots, N \\
\sum_{i=1}^{N} Q_{i}=1
\end{gathered}
$$

The spatial filling factor is derived from the participation ratio (or delocalization measure, $D$ ) which is a well known quantity in density matrix analysis, introduced by Bell and Dean [7] and Pipek [8] independently. The definition modified to the case of pixel intensities has the following form:

$$
D=\frac{1}{\sum_{i=1}^{N} I_{i}^{2}}
$$

$D$ shows the approximate number of filled lattice sites or pixels the distribution expands to. The spatial filling factor $(q)$ is the participation ratio divided by the total number of pixels $(N)$ :

$$
q=\frac{D}{N}
$$

The structural entropy $\left(S_{\text {str }}\right)$ is derived from the Shannon and Rényi entropies and was introduced by Pipek and Varga [9] to study the structure of many electron density functions. For pixel intensity distributions the Rényi entropies (the generalization of the Shannon entropy) are defined in the following way:

$$
S_{n}=\frac{1}{1-n} \log \left(\sum_{i=1}^{N} I_{i}^{n}\right)
$$

It can be proven that the Shannon entropy is the first member of the Rényi entropies. The Shannon entropy measures how much the pixel intensity distribution $\left(I_{i}\right)$ deviates from the uniform distribution (when all $I_{i}$ have the same value, then the image is homogeneous gray). The second Rényi entropy is the entropy of a uniform distribution over $D$ sites (also called the extension part of the Shannon entropy) and corresponds to the number of pixels with significantly high intensity. The difference between $S_{1}$ and $S_{2}$ characterizes the structure of the system, and it is called the structural entropy $\left(S_{s t r}\right)$ :

$$
S_{s t r}=S_{1}-S_{2}=S-\log D
$$

The structural entropy characterizes purely the deviation of the intensity distribution from the step function (which is in fact the black and white image).

The pair of functions $\left(q ; S_{s t r}\right)$ of the distribution $\left\{I_{i}, i=\right.$ $1, \ldots, N\}$ is called generalized localization, and it can be used for analyzing the topology free structure of the observed distribution. Our postulated new parameter, the localization factor is the value $(\alpha)$ which yields the smallest square error between the generalized localization of the AFM image and the generalized localization of the $\exp \left(-x^{\alpha}\right)$ probe function. In other words, it can be defined as the minimum of the $E(\alpha)$ error function based on (14).

$$
E(\alpha)=\left[S_{\text {str }}(q)_{\text {image }}-S_{\text {str }}(q)_{\exp \left(-x^{\alpha}\right)}\right]^{2}
$$

In the case of the AFM images, this exponent expresses the typical shape of hills and valleys of the topography maps. Two extremities of localization factor are $\alpha=0$ and $\alpha=\infty$ which correspond to totally flat surface and vertical-walled features, respectively.

To calculate the localization factor of an AFM image first the image is segmented to $k \mathrm{x} k$ number of smaller images. The value of $k$ should be selected taking into consideration 1) the scan size and resolution of the image, 2) the average size of the features on the image, in a way to guarantee, that the size of a segment is at least twice larger than the average size of the characteristic shapes (sampling criteria). The pair of functions $\left(q ; S_{s t r}\right)$ will then be calculated for all the segments, and $\alpha$ is determined as the minima of the $E(\alpha)$ error function calculated for this distribution. For more information please read the original articles regarding the introduction of the localization factor for AFM imaging $[4,6]$.

\section{EXPERIMENTAL}

\section{A. AFM Instrumentation}

AFM measurements were performed with a Veeco (lately Bruker) diInnova type scanning probe microscope (SPM). Contact mode measurements were done with ART D160 diamond probes. The sampling rate of the images presented in the paper is $512 \times 512$ obtained with $1 \mathrm{~Hz}$ scan rate. During the scans the PID values of the scanner feedback were optimized according to the Veeco User Manual to gain the best image quality. For data evaluation the Gwyddion 2.36 software was used [10].

\section{B. Nanoisland preparation}

Gold layers of a thickness between 14-16 nm were deposited onto four glass substrates via thermal evaporation at the Department of Experimental Physics, University of Debrecen. The layer thickness was measured after evaporation with a surface profiler. The subsequent thermal annealing of the four samples was done in a ceramic oven at $580{ }^{\circ} \mathrm{C}$ for four different time periods: $15 \mathrm{~min}, 30 \mathrm{~min}, 60 \mathrm{~min}$ and $120 \mathrm{~min}$, respectively. 


\section{RESUlTS AND DisCUSSION}

\section{A. Tip geometry and image reconstruction}

In this section we aim to demonstrate the effect of tip geometry on the image quality and the size of the nanoislands; to demonstrate the possibility to model/estimate the tip geometry and reconstruct/repair the image which was made with a blunt tip, and also, the ability of the localization factor parameter to help in the optimization of this surface reconstruction process. Fig. 2 presents a $25 \mu \mathrm{m}^{2}$ tapping-mode topography AFM image of gold nanoislands, prepared by thermal annealing the deposited 14-16 nm gold thin film for $120 \mathrm{~min}$. The image was obtained with a sharp, diamond AFM tip. As can be seen in Fig. 2, the nanoislands have sharp features and appear to be 'slender'.

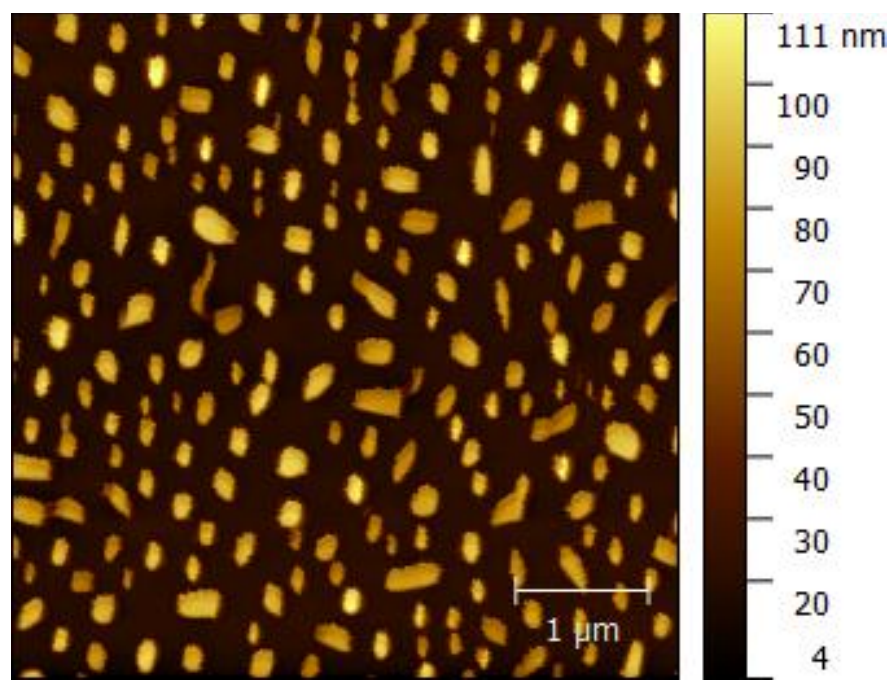

Fig. 2. Tapping-mode AFM topography image of gold nanoisland prepared by $120 \mathrm{~min}$ thermal annealing, measured with a sharp AFM tip. Image size: $5 \mu \mathrm{m}$ x $5 \mu \mathrm{m}$

To verify the sharpness of the tip and check whether an AFM image contains any convolution artifacts which would result from a blunt/damaged tip, the Gwyddion AFM post-processing software contains useful tools. By using the 'Tip->Blind Estimation' tool, one can run an algorithm on the whole image which aims to obtain the possible geometry of the tip, which was used for the imaging. The resulting tip geometry of this estimation algorithm used on the image of Fig. 2 is presented in Fig. $3 \mathrm{~A}$ and $\mathrm{B}$ (full iteration was used on the whole image, considering a tip of $17 \times 17$ pixels on the $512 \times 512$ image).

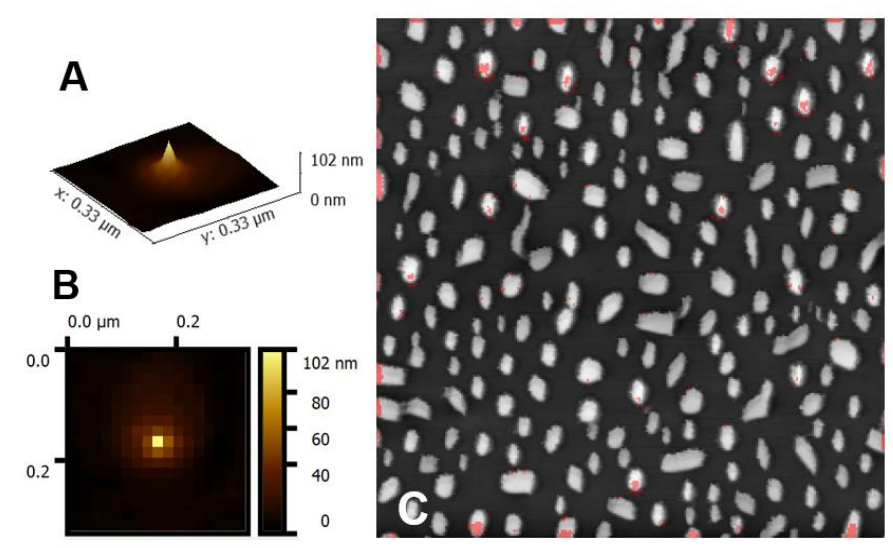

Fig. 3. A) 3D topography of the sharp tip used for the imaging. B) The same tip topography in 2D. The tip geometry was obtained by the Gwyddion 'Blind Estimation' function, using the whole image and 'Full iteration' with 17 pixels. C) 'Certainty Map' of Fig. 2 using the estimated sharp tip (red areas mark the uncertain regions).

As can be seen in Fig. 3, the estimated tip appears to be sharp. Looking at the 'Certainty Map' of Fig. 3/C, which was obtained by using this estimated tip geometry on the AFM image, we can say that our image is really free of possible tipbased artifacts. In the Certainty Map the uncertain areas are marked with red mask in Fig. 3 (note that the original output of the 'Certainty Map' function was inverted here for better visualization). In these points we can suspect, that the AFM tip touched the surface in multiple points instead of only one point, which means that there is a loss of information in these points.

Fig. 4 presents a $25 \mu^{2}$ tapping-mode topography AFM image of the same nanoislands sample, but measured with a blunt AFM tip. We can instantly see that - since the scan size of the images of Fig. 2 and 4 are the same - the size of the nanoislands are significantly larger. Observing more closely we can also see, that a pattern is repeated on the surface (note the small slot at the right side of nearly every nanoisland at their middle section), which clearly means tip convolution artifact and a blunt tip, which is possibly broken or contaminated. Performing the same tip estimation and certainty map operations on the image we can see the resulting tip geometries in Fig. 3. For our investigations the assumed tip size was increased gradually from 8 pixels to 15 pixels, the result of 10 and 13 pixels are presented in Fig. 5, as an illustration (the tip was estimated on a segmented image, containing only one nanoisland for the better estimation of the possible tip geometry). It can be seen, that the estimated tip is much blunter compared to Fig. 3, and also that increasing the assumed pixel number the estimated tip diameter increases accordingly. 


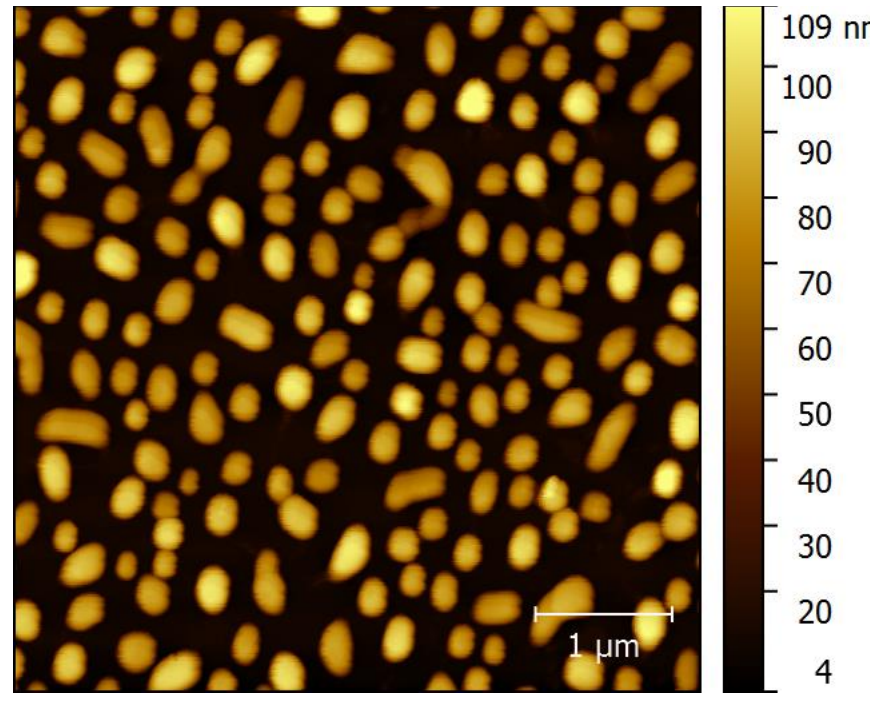

Fig. 4. Tapping-mode AFM topography image of gold nanoisland prepared by 120 min thermal annealing, measured with a blunt AFM tip. Image size: $5 \mu \mathrm{m}$ x $5 \mu \mathrm{m}$
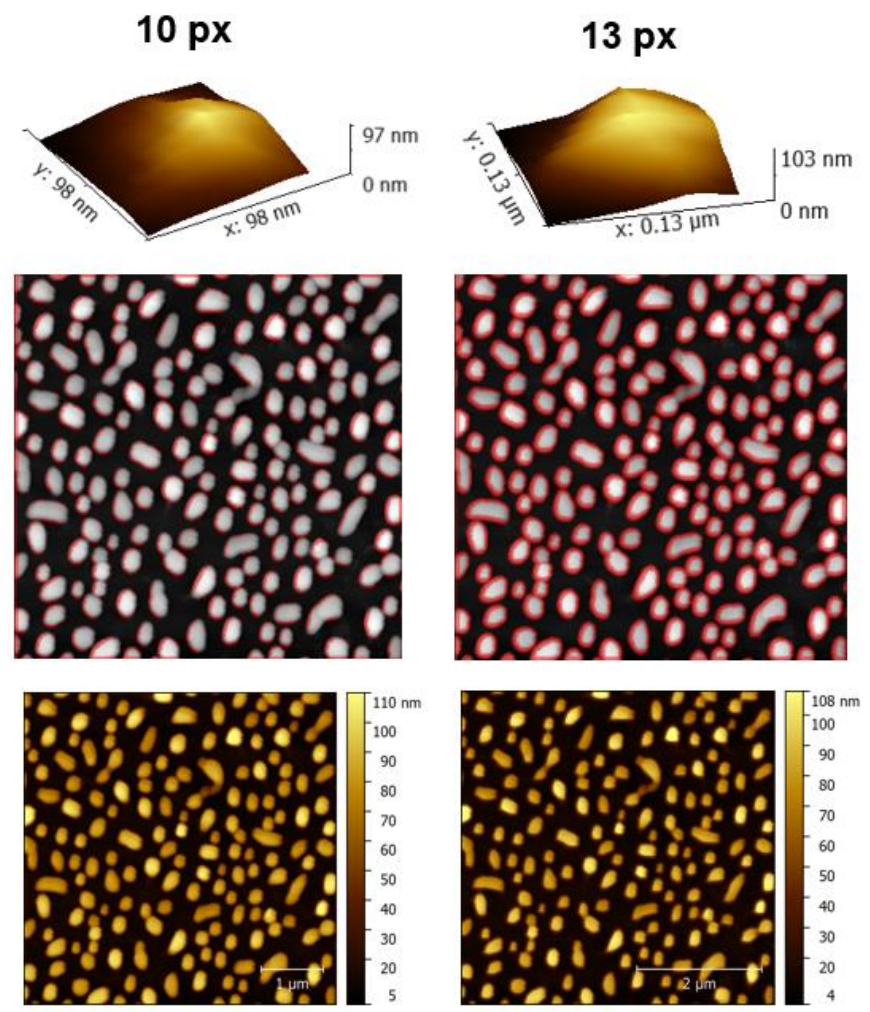

Fig. 5. Top row: 3D topography of the estimated blunt tip, using 'Blind Estimation' with 10 and 13 pixels on segmented images. Middle row:

'Certainty Maps' created by using the tips in the top row. Botton row: The topography image of Fig. 4 after 'Surface Reconstruction' using the estimated tip geometries.

The certainty maps confirm, that due to the blunt tip geometry, the side of the nanoisland are touched in multiple points, which resulted in the dilation of their size. By using the 'Tip -> Surface Reconstruction' function it is possible to try to reconstruct the image by applying a deconvolution algorithm with the estimated tip. This algorithm erodes the size of the particles, and the larger the estimated tip is, the more is the amount of erosion, as can be seen in the bottom row of Fig. 5 . Note that this surface reconstruction is only partial: the information which is missing from the points which are not touched by the tip cannot be regained by this deconvolution algorithm.

The biggest difficulty regarding the use of surface reconstruction is the precise estimation of tip geometry, since the estimation algorithm is very sensitive to input parameters, such as the assumed tip size. Depending only on the naked eye, it is impossible to precisely judge the amount of erosion which is required to properly reconstruct the surface, as can be seen on Fig. 5 for $10 \times 10$ and $13 \times 13$ pixel sizes. Is the former enough, or should we used an even bigger tip to reconstruct the image?

The function of the localization factor parameter, which was introduced in the Theory section is the quantitative characterization of the shape of the surface structures. Compared with the original image, the convoluted tip artifacts alter the shape of the surface structures, which can be detected by using this parameter. Besides, by using the segmentation algorithm for the determination of localization factor, as was introduced in our previous publications $[4,6]$, it will also be very sensitive to the surface ratio of the nanoparticles (total developed surface area of the nanoparticles / the scan size). Thus, the use of localization factor presents a possibility to characterize the effect of surface reconstruction, as demonstrated in Fig. 6.

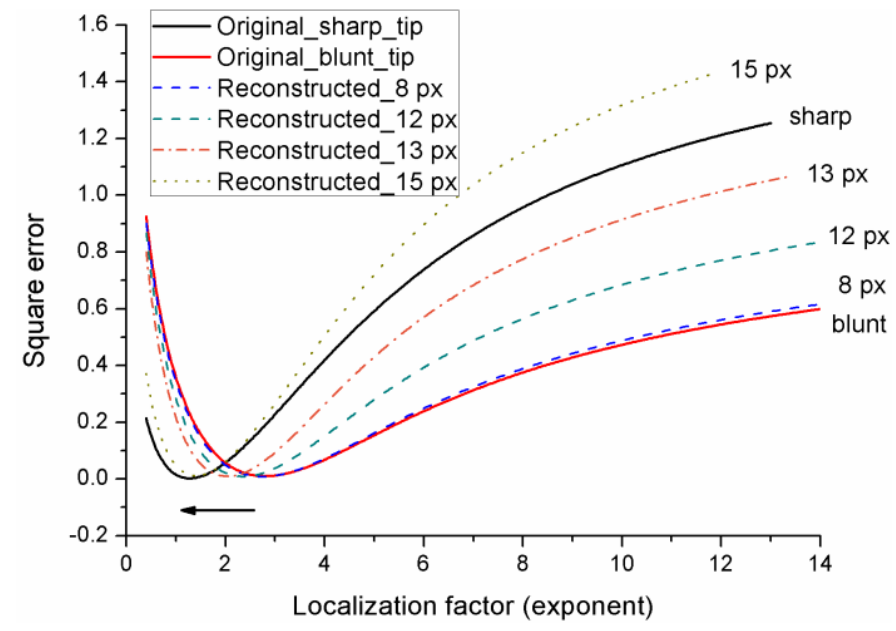

Fig. 6. Square error curves in funnction of the exponent of the probe function (localization factor). The arrow indicates the change in the localization factor (the minimum of the curves).

The curves in Fig. 6 are the error functions of Eq. (7) calculated for the AFM images and the $\exp \left(-x^{\alpha}\right)$ probe function. The minimum point of the curves is defined as the localization factor. It can be seen, that there is a clear difference between the curves obtained for the sharp tip (Fig. 2) and blunt tip (Fig. 4) images. The other curves were calculated on images reconstructed from the image made with the blunt tip and using estimated tip geometries with increasing assumed tip size (from $8 \times 8$ pixels to $15 \times 15$ pixels). The curves show, that by increasing the assumed tip size, and thus the amount of erosion during reconstruction, the localization factor shifts gradually in the direction of the image made with a sharp tip. Using the estimated tip geometry of $15 \times 15$ pixels the localization factor 
and the error function curve of the reconstructed image is nearly equal to the one obtained with the sharp tip, which means that this tip size is needed for the sufficient amount of erosion/reconstruction, in this example. Note, that although the two AFM images were obtained on the same sample but not on the exactly same area, the two curves would never completely fit. Also, as we previously noted it is never possible to perfectly reconstruct an AFM image due to the loss of information. Besides these notes, the localization factor clearly demonstrates the following three possibilities. If we have an AFM image from a sample which we can be sure that was measured with a sufficiently sharp tip, by obtaining the localization factor value for this image and comparing it with subsequent images from the same sample it is possible to 1) to detect, monitor and compensate tip wear/aging; 2) to decide whether other tips used to image the same sample were sufficiently sharp or not and 3) to help setting and optimizing the parameters of a surface reconstruction, as demonstrated in Fig. 6.

\section{B. Effects of image reconstruction on the characteristic surface parameters}

In this section we demonstrate the application of the described surface reconstruction method for the characterization of various nanoisland arrangements. AFM images were made on four samples, which were annealed for different time periods (15 $\mathrm{min}, 30 \mathrm{~min}, 60 \mathrm{~min}$ and $120 \mathrm{~min}$, respectively). The resulting AFM images are presented in Fig. 7. As can be seen, there is different amount of additive tip effect convoluted on the shape of the nanoislands, so surface reconstruction was applied on the images, as discussed before.
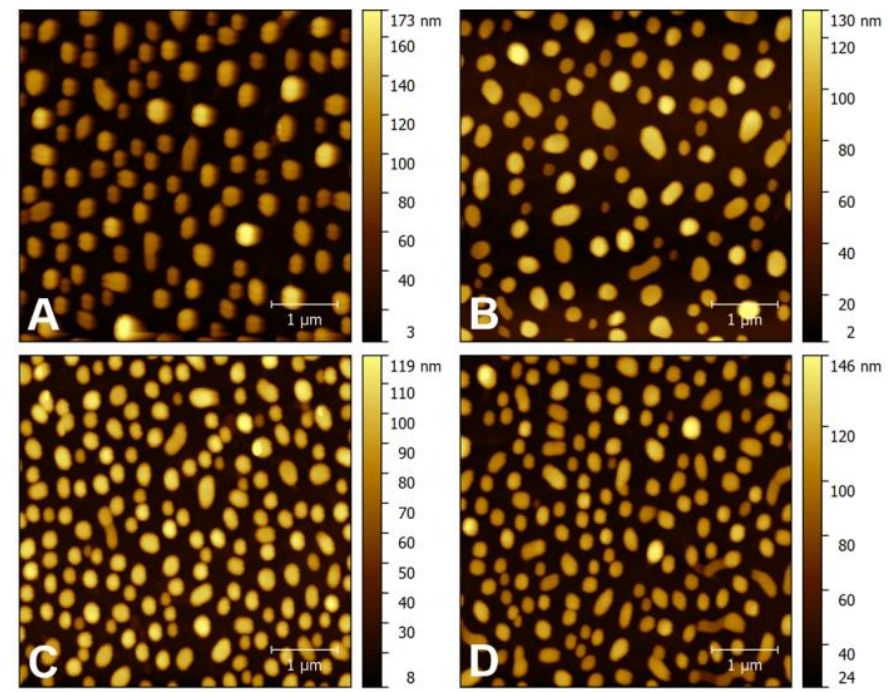

Fig. 7. Tapping-mode AFM topography images of gold nanoisland prepared by thermal anneling for various time periods. From A-D: $15 \mathrm{~min}, 30 \mathrm{~min}$, $60 \mathrm{~min}$ and $120 \mathrm{~min}$, respectively. The blunt tip was used for the imaging. Image size: $5 \mu \mathrm{m} \times 5 \mu \mathrm{m}$

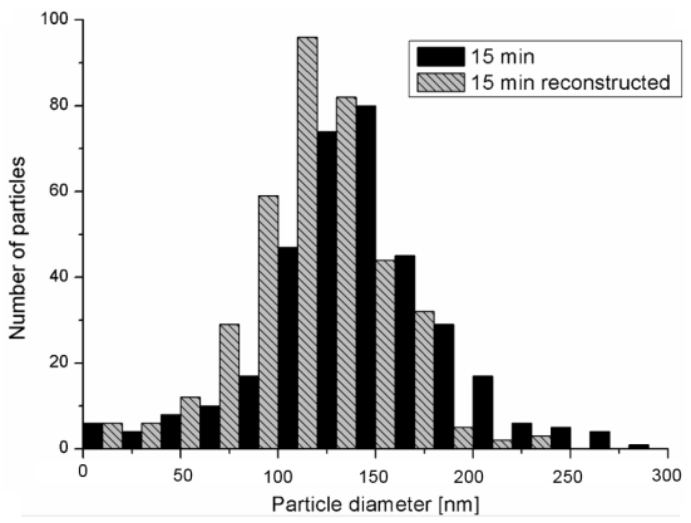

Fig. 8. Histograms of the equivalent spherical particle diameter of the nanoislands, obtained with the blunt AFM tip. Sum of three AFM images measured on the sample after 15 min annealing, before and after surface reconstruction.

Fig. 8 presents the distribution of the equivalent disc radius of the particles for the sample with 15 min annealing time, before and after surface reconstruction (the presented histograms are the sum of three AFM images). Fig. 9 compares the histograms based on the reconstructed images from the samples after $15 \mathrm{~min}$ and $60 \mathrm{~min}$ annealing.

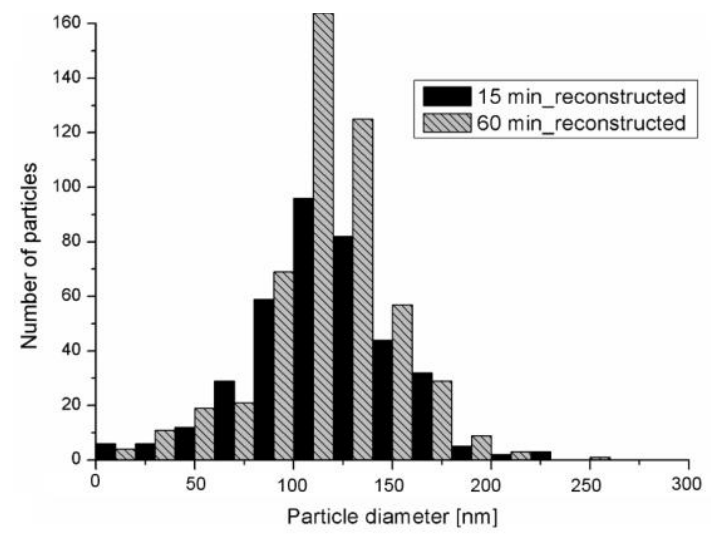

Fig. 9. Histograms of the equivalent spherical particle diameter of the nanoislands, obtained with the blunt AFM tip. Sum of three AFM images measured on the samples after 15 min and 60 min annealing, respectively, both after surface reconstruction.

The biggest difference between the samples with increased annealing times is in the number of particles. The majority of the particles falls into the 100-150 nm diameter range, based on the reconstructed images, as can be seen in the box charts of Fig. 10. As a result of the erosion the size of the particles decreases accordingly, which is confirmed by the presented distributions. 


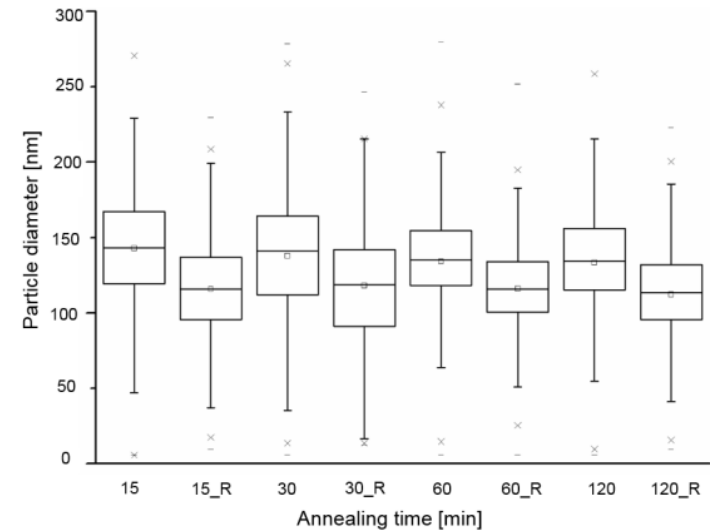

Fig. 10. Box charts illustrating the distribution of the equivalent particle diameters of the samples prepared with different annealing times. The obtained results after surface reconstruction are marked with ' $R$ '.

Figures 11-13 present three other important surface describing parameters which are greatly influenced by the quality of the tip and thus the surface reconstruction. The nanoisland density (Fig. 11) is calculated as the number of nanoparticles per unit scan area. With a blunt tip it is possible to merge small nanoparticles which are close to each other. By using proper deconvolution it is possible te separate these partially merged particles, which will be identified as separate particles. (In our case a simple height-tresholding was applied to identify the nanoparticles.) Hence, proper surface reconstruction increases the number of the recognized nanoparticles.

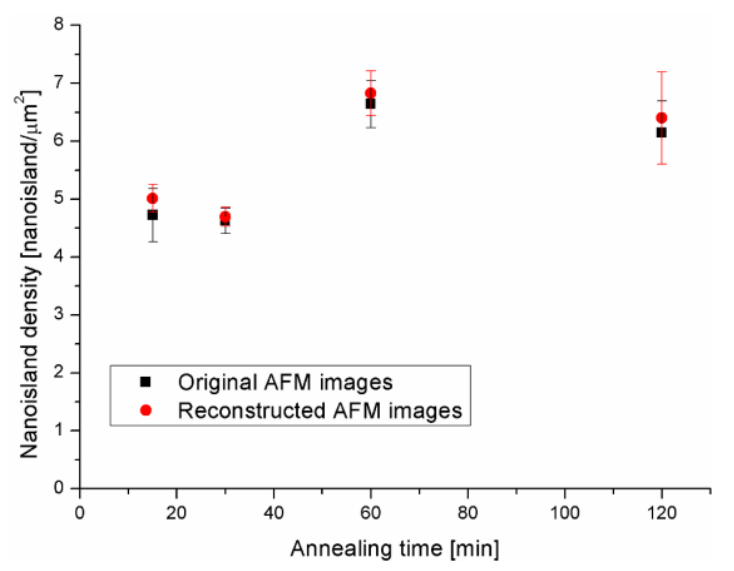

Fig. 11. Nanoisland densities calculated based on three AFM images made on the samples before and after surface reconstruction.

In the same way, as a result of the erosion, the surface ratio of the particles (total nanoparticle developed area / scan area) decreases significantly (see Fig. 12). Since the density of the nanoisland, more specifically, the size and distance between them strongly affect the coupled plasmon resonance between them, the precise estimation of these parameters are important for proper surface characterization.

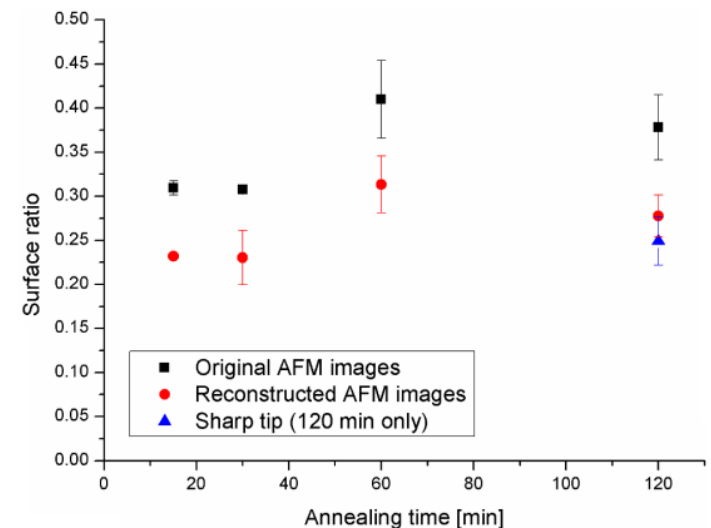

Fig. 12. Surface ratios calculated based on three AFM images made on the samples before and after surface reconstruction.

Fig. 13 presents the localization factor values measured on the AFM images with and without surface reconstruction. The deconvolution algorithm decreased the localization factor values with 0.4-0.6 depending on the sample. It can be observed, that the localization factor parameter strongly correlates with the previous parameters (the higher the surface ratio or density of the particles, the higher the localization factor). The reason for this, is that the current image segmentation method does not filter out the flat background around the particles, and the amount of these dark areas on the image strongly contribute to the obtained localization factor values. (In this work the images were segmented to $5 \times 5$ pieces, for the optimization of this segmentation, see our previous publication [6].) We are currently working on a new segmentation method to detect and cut only the shape of the nanoislands and use them as segments for the localization factor calculations, and thus the obtained values would only include the shape of the particles, which would be its original purpose.

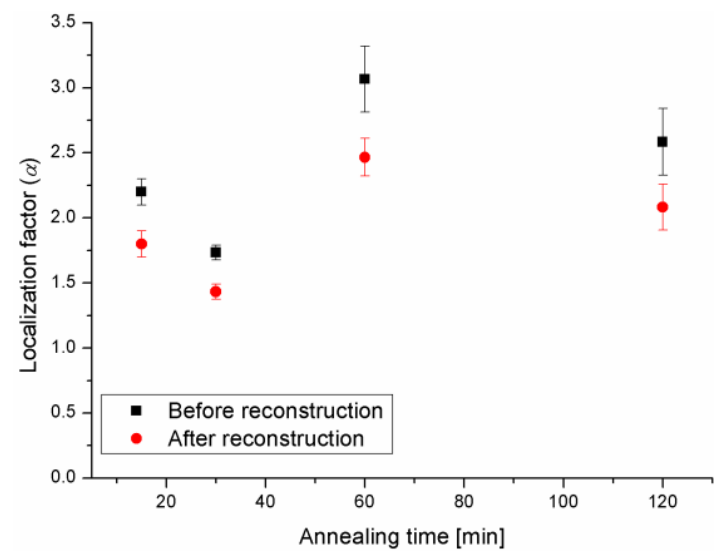

Fig. 13. Localization factor values obtained on the nanoisland arrangements, before and after surface reconstruction. (Calculated for three images per sample).

Note that the areas used for AFM imaging was $25 \mu \mathrm{m}^{2}$, which is small compared to the whole $2 \mathrm{~cm}^{2}$ area of the samples. Although different areas on the surface were investigated, it is still not possible to completely cover the whole surface and local inhomogeneities could contribute to the observed differences. 
2016 IEEE $22^{\text {nd }}$ International Symposium for Design and Technology in Electronic Packaging (SIITME)

\section{CONCLUSIONS}

Gold nanoisland arrangements were prepared by thermal annealing of pre-deposited gold thin films on glass substrates and were characterized with atomic force microscopy. A common problem concerning the AFM imaging of nanoparticles, namely the tip convolution effect, was studied in detail. It was demonstrated that the localization factor parameter can be used both to assess the possibility of tip effects and also to control the parameters of surface reconstruction via tip deconvolution. The effect of surface reconstruction for various nanoisland arrangements was studied and its importance for obtaining reliable characteristic surface parameters was discussed.

\section{ACKNOWLEDGMENT}

The research leading to these results has received funding from the ProProgressio foundation. Attila Bonyár is grateful for the support of János Bolyai Scholarship.

\section{REFERENCES}

[1] Xiaodong Cao, Yongkang Ye, Songqin Liu "Gold nanoparticle-based signal amplification for biosensing", Anal. Biochem., Vol. 417, pp. 1-16, 2011.
[2] Pengxiang Zhao, $\mathrm{Na} \mathrm{Li}$, Didier Astruc "State of the art in gold nanoparticle synthesis", Coord. Chem. Rev., Vol. 257, pp. 638- 665, 2013.

[3] A. Serrano, O. Rodríguez de la Fuente, M. A. García, "Extended and localized surface plasmons in annealed Au films on glass substrates", J. App. Phys., Vol. 108, pp. 074303, 2010.

[4] A. Bonyár, M. L. Molnár, G. Harásnyi, " Localization factor: A new parameter for the quantitative characterization of surface structure with atomic force microscopy (AFM) ", Micron, Vol. 43, Issues 2-3, pp. 3053010,2012

[5] A. Bonyár, P. Lehoczki, "An AFM study regarding the effect of annealing on the microstructure of gold thin films ", Proc. Of the 36th IEEE-ISSE International Spring Seminar on Electronics Technology, Alba Iulia, Romania, pp. 317-322, 2013.

[6] A. Bonyár, "AFM characterization of the shape of surface structures with localization factor", Micron, Vol. 87. pp. 1-9, 2016.

[7] R. J. Bell, P. Dean, "Atomic vibrations in vitreous silica", Discuss. Faraday Soc., pp. 50-55, 1970.

[8] J. Pipek, "Localization measure and maximum delocalization in molecular systems", Int. J. Quantum Chem., Vol. 36, pp. 487-501, 1989.

[9] J. Pipek, I. Varga, "Universal classification scheme for the spatiallocalization properties of one-particle states in finite, d-dimensional system", Phys Rev A., Vol. 46, 3148-3163, 1992.

[10] D. Nečas, P. Klapetek, "Gwyddion: an open-source software for SPM data analysis", Cent. Eur. J. Phys. Vol. 10, Issue 1, pp. 181-188, 2012. 